\title{
Mediation vs pseudomediation in kindergarten children
}

DONALD H. KAUSLER and JOHN W. DEICHMANN, Saint Louis University, St. Louis, Mo. 63103

Mediated positive transfer for kindergarten children was tested by means of the $A-B, B-C, A-C$ paradigm, with all three stages being experimentally acquired in immediate succession. Additional conditions were included to control for the occurrence of pseudomediation as a possible confounding factor in mediational transfer conditions. The evidence supported the occurrence of mediated positive transfer in young children. No evidence was found for the presence of pseudomediation.

Mediated transfer in children as young as kindergarten age has been demonstrated (e.g., Norcross \& Spiker, 1958) with tasks that assume links from natural language habits. There has been, however, little investigation of mediated transfer in children with tasks that only involve experimentally acquired links (Goulet, 1968). One concern of the present study was to test for the presence of mediated facilitation in young children (kindergarten level) by means of the A-B, B-C, A-C forward chaining paradigm (Horton \& Kjeldergaard, 1961) when all three stages are experimentally acquired.

An important related issue in testing for mediated facilitation at any age level is the possible presence of what Mandler \& Earhard (1964) have labeled pseudomediation. Pseudomediation refers to the fact that the A-B and A-C stages of mediational paradigms enter into the classical competitioninterference relationship of negative transfer and the fact that the amount of such interference during third list practice is less in the experimental condition receiving A-B, B-C prior lists than it is in the control condition receiving A-B, D-C prior lists. That is, the manifestation of positive transfer for A-C experimental pairs, relative to A-C control pairs, is presumed to be an artifact that actually reflects a difference in overall negative transfer effects for the two conditions. Mandler and Earhard identified the likely causal mechanism for pseudomediation to be a competition-unlearning relationship generated during $\mathrm{B}-\mathrm{C}$ or second list practice by experimental Ss. This relationship results from the A-B, A-C arrangement formed by the R-S or "backward" associations of the first list (i.e., B-A associations) and the S-R or "forward" associations of the second list (i.e., B-C associations). Unlearning of first list $A-B$ associations are thus less available for interference during practice on third list A-C pairs. A comparable source of unlearning of A-B associations during second list practice is not present during second list practice for control Ss, and competing $\mathrm{A}-\mathrm{B}$ associations are presumed to exert their interfering effect in full strength during third list practice.

The commonly held view (e.g., White, 1964) that young children are especially prone to interference effects suggests that pseudomediation may be an important phenomenon to take into account in interpreting mediated transfer effects for children, especially for children at the kindergarten level. Consequently, the present study included the appropriate test for pseudomediated transfer as proposed by Mandler \& Earhard (1964). This test involves a contrast between third list performance for an experinental group receiving an A-B, B-C, A-E list sequence and a control group receiving an A-B, D-C, A-E sequence. The experimental condition provides for the possible reduction of interference on the third list, as in the A-B, B-C, A-C sequence, but without the opportunity of simultaneously employing a mediating A-B-C chain, as in the latter condition.

\section{Subjects and Design}

The Ss were 56 children who were students in kindergarten classes of a middle-class, suburban public school system. Seven additional Ss were eliminated from the study for failure to follow instructions. The 56 Ss ranged in age from 65 to 79 months, with a mean age of 73.78 months. They were assigned alternately, by sex, to the four conditions of the study. These conditions were a standard mediational experimental condition (Group ME), receiving A-B, B-C, A-C lists, a standard mediational control condition (Group $\mathrm{MC}$ ), receiving A-B, D-C, A-C lists, a pseudomediational experimental condition (Group PE), receiving A-B, B-C, A-E lists, and a pseudomediational control condition (Group $\mathrm{PC}$ ), receiving $\mathrm{A}-\mathrm{B}, \mathrm{D}-\mathrm{C}$, A-E lists. Thus the design was a single-variable, four-level design that permitted multiple comparisons between Groups $\mathrm{ME}$ and MC and Groups PE and PC.

Lists

Each paired-associate list was composed of five stimulusresponse pairs. The items of these pairs were black and white line drawings taken from the Peabody Picture Vocabulary Test. Twenty-five such items were assigned randomly to five subsets, A, B, C, D, and E, of five items each. From these subsets the five pairs of each list were prepared for each of the four groups of Ss. All 56 Ss received A-B (i.e., items from the A subset were stimulus elements, and items from the B subset were response elements) pairs as the first list. For purposes of greater generalization of results two different list contents were employed as second and third lists for each of the four groups. Half of the Ss in Group ME received B-C pairs (i.e., items from the B subset as stimuli, etc.) in the second list and A-C pairs in the third list; the other half received B-E pairs in the second list and A-E pairs in the third list. Similarly, the two list contents for second and third lists were D-C, A-C, and D-E, A-E for Group MC, B-C, A-E, and B-E, A-C for Group PE, and D-C, A-E, and D-E, A-C for Group PC. Thus the four groups were equated in terms of receiving identical pairings (half of each group receiving $A-C$ pairs and half receiving $A-E$ pairs) on the critical third list. The three list sequence conforms to the appropriate mediational, mediational control, pseudomediational, or pseudomediational control paradigm for each S. The two specific list contents entering into the second and third lists were subsequently found to be unrelated to contrasts between the relevant paradigms, and list content was omitted from all subsequent statistical analyses.

Procedure

Prior to the introduction of the learning tasks each $S$ was shown the pictures, one at a time and in a random order, used in the transfer lists as stimuli and responses and was asked to name the object depicted. This enabled $E$ to make certain that the items were in S's vocabulary.

The learning tasks were introduced by means of paired-associate instructions modified to be comprehended by young children and accompanied by several trials on a two-pair practice list. The items in the lists per se were prepared on slides and projected on a small telescreen by means of a Carousel projector. The anticipation method was used, with each stimulus item being exposed alone for $5 \mathrm{sec}$, followed by the simultaneous exposure of the stimulus with its paired response item for $5 \mathrm{sec}$. Three different random orders of presenting pairs were employed for each list as a control over possible serial learning effects. The intertrial interval was 10 sec for each list. Approximately $10 \mathrm{sec}$ separated first-second and second-third list practice, with $\mathbf{S}$ being informed during this period that he would have additional pairs to learn. 
Practice on each list continued to a criterion of two perfect trials which were not necessurily consecutive.

\section{RESULTS AND DISCUSSION}

Stage 1

The mean and standard deviation for trials to criterion on the first list were 5.43 and 1.91 for Group ME, 5.79 and 1.93 for Group MC, 6.86 and 2.14 for Group PE, and 7.07 and 1.98 for Group PC. An analysis of variance revealed a between-group effect that approached statistical significance $(\mathrm{F}=2.28, \mathrm{df}=3 / 52, \mathrm{p}<.10)$. Thus the possibility exists that the four groups differed in initial learning proficiency, a difference that requires a cautious interpretation of performances on the two transfer lists. However, the critical contrasts in transfer performance are those between Groups $\mathrm{ME}$ and $\mathrm{MC}$ and Groups $\mathrm{PE}$ and PC. Orthogonal analyses of first list scores indicated that in both cases the two groups were equated in learning proficiency (Group ME vs Group MC, $\mathrm{F}<1$, df $=1 / 52$; Group PE vs Group $P C, F<1$, df = 1/52), and subsequent contrasts on second and third lists are unlikely to be confounded by differences in initial proficiency.

Stage 2

As noted earlier, Groups $\mathrm{ME}$ and $\mathrm{PE}$ represent negative transfer paradigms, relative to Groups $\mathrm{MC}$ and $\mathrm{PC}$, to the extent that first list R-S associations compete with second list $\mathrm{S}-\mathrm{R}$ associations. For trials to criterion on the second list the mean and standard deviation were 6.71 and 2.97 for Group ME, 6.50 and 1.87 for Group MC, 8.79 and 2.94 for Group PE, and 8.07 and 2.23 for Group PC. Surprisingly, all four groups required more trials to reach criterion on the second list than on the first list, in spite of the overall positive transfer expected in each group through nonspecific sources such as learning-to-learn.

Both Group ME and Group PE required slightly more trials to reach criterion than did their respective control groups. Although the overall analysis of variance revealed that the between-group effect approached significance $(F=2.59$, $\mathrm{df}=3 / 52, \mathrm{p}<.10$ ), both the Group ME vs Group MC and the Group PE vs Group PC orthogonal comparison fell far short of significance (both $\mathrm{Fs}<1$, $\mathrm{df}=1 / 52$ ). The failure to find negative transfer in the $A-B, B-C$ paradigm with kindergarten Ss may be contrasted with the well-established (e.g., Murdock, 1958) finding of negative transfer with college-age Ss. However, a complicating factor in abrogating the presence of specific negative transfer in the present study is the fact that the list content for the experimental and control conditions differed (Groups ME and PE received either B-C or B-E pairings, whereas Groups $\mathrm{MC}$ and $\mathrm{PC}$ received either $\mathrm{D}-\mathrm{C}$ or D-E pairings), and the possibility that the pairings may have varied in difficulty cannot be ruled out. The need to replicate the present conditions with list content equated on the second list rather than on the first list seems apparent.

Stage 3

The mean and standard deviation for trials to criterion on the third list were 4.64 and 1.50 for Group ME, 8.00 and 3.68 for Group MC, 7.79 and 2.45 for Group PE, and 7.86 and 3.01 for Group PC, and the between-group effect of the overall analysis of variance was significant $(F=4.77, \mathrm{df}=3 / 52$, $\mathrm{p}<.01)$. As is apparent from the above means, the between-group effect is attributable solely to the facilitation evident in Group ME vs to each of the other three groups (Scheffe's test; comparison of Group ME vs Groups MC, PE, and $\mathrm{PC} ; \mathrm{F}=14.26, \mathrm{df}=3 / 52, \mathrm{p}<.01)$. An additional comparison revealed a superior performance for Group ME relative to its control condition (Group MC) (Scheffé's test; $F=10.22, \mathrm{df}=3 / 52, \mathrm{p}<.05)$. In view of the pronounced difference in variability for Groups $M E$ and $M C$, the difference between them in third list performance was also tested by means of the median test, with the outcome again reaching statistical significance $\left(\chi^{2}=5.17, \mathrm{df}=1, \mathrm{p}<.05\right)$. On the other hand, the comparison between Groups PE and PC fell short of statistical significance $(F<1)$.

The analyses reported above provide convincing evidence for the presence of mediated transfer in kindergarten children. The fact that the mediated facilitation demonstrated in Group ME cannot be reasonably attributed to pseudomediation is strongly supported by the failure to find a significant difference between Groups $\mathrm{PE}$ and $\mathrm{PC}$, the groups introduced in the present design to serve as an indication of the extent of pseudomediation. In fact, the occurrence of pseudomediation, as defined by Mandler \& Earhard (1964), remains to be demonstrated. Further support for these conclusions may be gathered by an analysis of the total number of correct anticipations across the first three trials on the third list. The mean and standard deviation were 10.43 and 2.79 for Group ME, 7.71 and 3.99 for Group MC, 5.57 and 2.41 for Group PE, and 5.93 and 3.22 for Group PC. The significant between-group effect $(F=6.92, \mathrm{df}=3 / 52, \mathrm{p}<.01)$ was again attributable to the Group ME vs Groups MC, PE, and $\mathrm{PC}$ comparison (Scheffé's test; $\mathrm{F}=17.05, \mathrm{df}=3 / 52$, $p<.01$ ), and again there was no reliable evidence for the occurrence of pseudomediation (Group PE vs Group PC contrast; $\mathrm{F}<1$ ).

\section{REFERENCES}

GOULET, L. R. Verbal learning in children: Implications for developmental research. Psychological Bulletin, 1968, 69, 359-376.

HORTON, D. L., \& KJELDERGAARD, P. M. An experimental analysis of associative factors in mediated generalization. Psychological Monographs, 1961, 75, Whole No. 515.

MANDLER, G., \& EARHARD, B. Pseudomediation: Is chaining, an artifact? Psychonomic Science, 1964, 1, 247-248.

MURDOCK, B. B., JR. "Backward" associations in transfer and learning. Journal of Experimental Psychology, 1958, 55, 111-114.

NORCROSS, K. J., \& SPIKER, C. C. Effects of mediated associations on transfer in paired-associate learning. Journal of Experimental Psychology, 1958, 55, 129-134.

WHITE, S. H. Evidence for a hierarchical arrangement of learning processes. In C. C. Spiker and L. P. Lipsitt (Eds.), Advances in child development and behavior. Vol. 2. New York: Academic Press, 1964. 